\title{
Does Duration of Spousal Caregiving Affect Risk of Depression Onset? Evidence from the Health and Retirement Study
}

\author{
Benjamin D Capistrant, ScD ${ }^{1}$, Lisa F. Berkman, $\mathrm{PhD}^{2,3,4}$, and M. Maria Glymour, $\mathrm{ScD}^{2}$ \\ ${ }^{1}$ Carolina Population Center, University of North Carolina - Chapel Hill \\ ${ }^{2}$ Department of Society, Human Development and Health, Harvard School of Public Health \\ ${ }^{3}$ Department of Epidemiology, Harvard School of Public Health \\ ${ }^{4}$ Harvard Center for Development and Population Studies, Harvard University
}

\begin{abstract}
Objectives-To assess the association of current and long-term spousal caregiving with risk of depression in a nationally (U.S.) representative sample of older adults.

Methods-We followed married and depression-free Health and Retirement Study (HRS) respondents aged 50+, $(\mathrm{n}=10,105)$ at baseline, from 2000 to 2010 . Current ( $\geq 14$ hours/week of I/ADL help for a spouse in the most recent biennial survey) and long-term caregiving (care at two consecutive surveys) were used to predict onset of elevated depressive symptoms ( 23 on a modified CES-D scale) with discrete-time hazards models and time-updated exposure and covariate information.
\end{abstract}

Results-Current caregiving was associated with significant elevations in risk of depression onset (HR=1.64; Wald $\left.\chi^{2}, 1 \mathrm{df}: 28.34, \mathrm{p}<0.0001\right)$. Effect estimates for long-term caregiving were similar (HR=1.52, Wald $\chi^{2}$, 1df: 3.63, $\left.\mathrm{p}=0.06\right)$.

Conclusions-Current spousal caregiving significantly predicted onset of depression; the association was not exacerbated by longer duration of caregiving.

\section{Key Terms}

caregiving; spouses; depressive symptoms; depression; older adults

\footnotetext{
(C) 2013 American College of Cardiology Foundation. Published by Elsevier Inc. All rights reserved.

Corresponding Author: Benjamin D Capistrant, Carolina Population Center, University of North Carolina - Chapel Hill, CB 8120, University Square, 123 West Franklin St, Chapel Hill, NC 27516 USA Telephone: 919-966-5891, Fax: 919-966-6638, ben_capistrant@unc.edu.

Publisher's Disclaimer: This is a PDF file of an unedited manuscript that has been accepted for publication. As a service to our customers we are providing this early version of the manuscript. The manuscript will undergo copyediting, typesetting, and review of the resulting proof before it is published in its final citable form. Please note that during the production process errors may be discovered which could affect the content, and all legal disclaimers that apply to the journal pertain.

Previous Presentation: Portions of this paper were presented at the Population Association of America 2011 Annual Meeting in Washington, DC on March 31, 2011.

Conflicts of Interest: None to declare.

List of Supplemental Digital Content:

Supplemental Digital Content 1: CGDEP_SDC_Table A_Revised_20120926.docx
} 
Caregiving is common among older age spouses and has long been associated with increased depressive symptoms for the caregiver. Recent meta-analyses of articles on caregivers and depression ${ }^{1}$ found spousal status and number of months as a caregiver were significantly associated with caregiver depression. However, evidence is in fact quite mixed $^{2}$ and many of the articles comprising the literature base have methodological limitations, including small, convenience samples and cross-sectional designs. We used the U.S. Health and Retirement Study to examine whether current or longer-term caregiving for a spouse predicted onset of depression in a nationally representative sample of older adults. We posited both current and long-term caregiving would be positively associated with risk of incidence of elevated depression symptoms; that long-term caregiving would pose higher risk of incidence of elevated depressive symptoms than current caregiving.

\section{Methods}

HRS is a longitudinal survey of a national sample of US adults aged 50+ years and their spouses. Details of the study are provided elsewhere. ${ }^{3}$ Enrollments occurred in 1992, 1993, or 1998 with biennial interviews through 2010. The most up to date follow-up information shows retention above $80 \%$ through 2008 . HRS was approved by the University of Michigan Health Sciences Human Subjects Committee and these analyses were determined exempt by Harvard School of Public Health Office of Human Research Administration.

This study was restricted to married HRS participants born 1900 to 1947 and interviewed in 2000 , which was the earliest year when caregiving assessments were consistently worded and asked with respect to spouses. The HRS sample included 11,476 age-eligible, married respondents interviewed in 2000. We excluded 1,885 (16.4\%) who reported a prior elevated symptoms of depression in 2000 and 171 (1.5\%) who were missing key covariate information, leaving 9,420 individuals contributing person-time to the primary analyses.

Spousal caregiving demand was calculated in each biennial interview wave (2000-2008), based on the care recipient's report regarding assistance with activities of daily living (ADLs) and instrumental activities of daily living (IADLs). HRS respondents with care needs were asked to list the people who most provided assistance in the last month and, for each person, the estimated time spent on caregiving each day. An indicator variable was generated for whether a spouse was a listed caregiver and we calculated amount of care provided per week for these analyses. To be consistent with other studies of caregiving using these data ${ }^{4}$ the primary exposure classification was a dichotomized variable of $<14$ and $\geq 14$ hours of care per week. Long-term caregiving was defined as providing $\geq 14$ hours of care per week during two consecutive biennial survey assessments; this exposure was calculated between 2002-2008.

Depressive symptoms were self-reported by the caregiver using a modified, 8-item version of the Centers for Epidemiologic Studies-Depression (CES-D) scale. Our outcome variable was a dichotomized indicator for whether the respondent reported $\geq 3$ depressive symptoms in the past two weeks. This threshold was previously found to provide high sensitivity and specificity for depression per the CIDI-SF. ${ }^{5}$ 
The HRS survey design includes both individuals in a couple (e.g., both husband and wife) as survey respondents. There are a number of possibilities of combinations of caregiver/ care-recipient relationships within one couple; both, only one, or neither respondents in the household could be caregivers and care recipients. Since not everyone in the sample is a caregiver or care recipient, we also refer throughout to the unit of analysis as "respondents"; we refer to their spouse as "spouse" where necessary. In our main analyses, for both current and long-term caregivers, we compare respondents who are caregivers to a reference group of all other respondents; for simplicity, we refer to the covariates in these analyses as "caregiver" and "care recipient" characteristics to refer to respondent and spouse's characteristics, respectively. In our sensitivity analysis, we reasoned that because one provides care only when your spouse needs care, the "potential caregivers" should be restricted to individuals whose spouses had care needs. We therefore restricted the sample to respondents whose spouses have care needs (e.g., a wife is included as a respondent only if her husband has a care need); thus, this analysis compares caregiver respondents to "potential" caregiver respondents, rather than to all respondents, adjusting for the caregiver/ "potential" caregiver's and the "potential" care recipient's characteristics, respectively.

Covariate information was self-reported; caregiver demographic, SES, and health information was reported by the caregiver and care recipient health information was reported directly by the care recipient. Variables defined at baseline included caregiver's demographics: age and age-squared; race; Hispanic ethnicity; gender; length of marriage; and socioeconomic position: years of education ( $0-17)$; father's education (categorical, $<8$ /

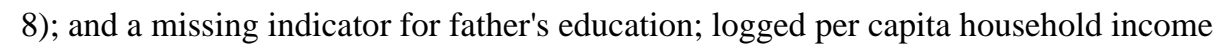
(baseline. Time-varying covariates were lagged one wave behind exposure assessment and included: caregiver health status: current drinking (any/none in past two weeks), current smoking status (yes/no), a summary of total number of self-reported conditions (high blood pressure, diabetes, cancer, lung disease, heart disease, stroke, psychiatric problems, and arthritis) calculated in the RAND version of the HRS dataset, and self-rated health (excellent/very good/good v. fair/poor); and care recipient health status: summary score of chronic conditions, care recipient self-rated health, and care recipient self-report of doctor's diagnosis of a memory illness. For observations with missing values, we set continuous variables to the mean and categorical variables to the referent group and included missing indicator variables in the analytic models.

We used discrete-time hazard models to test our three hypotheses. We conducted analyses using SAS 9.2 with PROC GENMOD using a logit link, and robust variance estimates, and weights as described below. The outcome was elevated self-reported caregiver depressive symptoms, and individuals were censored at death, marriage dissolution, or first onset of elevated depressive symptoms. We present unadjusted hazard ratios, and adjusted HRs that include the groups of covariates defined above sequentially with respect to their likely temporal ordering. We adjusted for time-varying confounding, loss-to-follow-up, and survivor bias by using inverse probability of treatment weights. Stabilized weights were calculated using previously described protocols ${ }^{6}$ and we truncated the top percentile of the weights at the value of 99th percentile to address the weights' skewed distribution. Also, we conducted sensitivity analyses of the association between caregiving and elevated depressive symptoms only among respondents whose spouses had care needs (referred to as "potential 
care recipients"). We applied the HRS sampling weights to make the population representative of the 2000 US population aged 50+ years.

\section{Results}

There was an average of 5.7 years of follow-up. There were 2,363 events of elevated depressive symptoms over 27,074 person-waves (wave 2 years) of follow-up among current caregivers and 1,456 events over 19,629 person-waves of follow-up among long-term caregivers. Over all person-years of follow-up, 3.2\% (person waves ( $\mathrm{PW}=877$ ) were classed as current caregivers and $1.2 \%(\mathrm{PW}=226)$ were classed as long-term caregivers; $10.8 \%$ $(\mathrm{PW}=2917)$ of the total observations were included in the sensitivity analysis restricted to spousal pairs with a prevalent care need.

We examined crude and adjusted estimates of caregiving risk on depression (Table 1). The crude association between caregiving and risk of elevated depressive symptoms was over two-fold for current caregivers (HR=2.10; 95\% CI: 1.71, 2.58; Wald $\chi^{2}, 1 \mathrm{df}: 49.38$, $\mathrm{p}<0.0001)$ and long-term caregivers (HR=1.92; 95\% CI: 1.27, 2.89; Wald $\chi^{2}, 1 \mathrm{df}: 9.6$, $\mathrm{p}=0.002$ ). Once adjusted and weighted for all covariates, current caregiving was significantly associated with risk of elevated depressive symptoms (HR=1.64; 95\% CI: 1.32 , 2.04; Wald $\chi^{2}, 1 \mathrm{df}: 28.34, \mathrm{p}<0.0001$ ). In the fully adjusted model, long-term caregiving was associated with a HR of 1.52 for elevated depressive symptoms, but this estimate was not statistically significant (95\% CI: 0.98, 2.35; Wald $\chi^{2}, 1 \mathrm{df}: 3.63$, $\left.\mathrm{p}=0.06\right)$. We found no significant differences in estimated associations between caregiving measures and risk of elevated depressive symptoms across gender, race, education, or recipient cognitive status (see Table A, Supplemental Digital Content 1).

In analyses restricted to respondents in households with a potential care recipient, fullyadjusted estimates were attenuated with wide CIs that included the null for both current $\left(\mathrm{HR}=1.21 ; 95 \% \mathrm{CI}: 0.91,1.61\right.$; Wald $\left.\chi^{2}, 1 \mathrm{df}: 1.79, \mathrm{p}=0.18\right)$ and long-term caregivers (HR=1.01; 95\% CI: 0.64, 1.59: Wald $\chi^{2}, 1 \mathrm{df}: 0.00, \mathrm{p}=0.97$; Table 1).

\section{Conclusion}

This study found that current spousal caregiving predicted an increased risk of onset of elevated depressive symptoms. However, we find no support for our hypothesis that longer duration of caregiving poses higher risks of onset of elevated depressive symptoms than shorter spells of caregiving. Current and long-term caregiving were associated with similar magnitudes of risk of elevated depressive symptoms.

These results are consistent with prior research on caregiving and depression. Several empirical studies have found no association between duration of caregiving and caregiver mental health ${ }^{7}$, including a longitudinal study that found duration of caregiving was not a significant predictor of caregiver's depressive symptoms. ${ }^{8}$ This pattern may be explained by findings from Townsend et al's longitudinal study comparing alternative theoretical models for how duration of care duties affects the caregivers. ${ }^{9}$ Their data did not support a "wearand-tear" hypothesis that caregivers have continuously worsening health over the duration of their care duties. 
Limitations of our study include reliance on self-reported data and a brief CES-D for assessment of depressive symptoms. ${ }^{5}$ We have no diagnostic measure of depression. This measure may not be as sensitive to someone whose mood is persistently low as it is to a larger change in the number of depressive symptoms. In HRS, the care recipient, not the caregiver, reported the amount of care provided, which may have generated differential misclassification bias. This limitation is weighed against the potential advantages of avoiding same-source bias for reporting of the exposure and the outcome. Also, the wide CIs suggest we were underpowered to detect moderate associations between long-term caregiving and onset of depression.

The most important limitations we faced are common to nearly all studies of caregiving: we cannot disentangle the mental effects of having a loved one who needs care from the effects of providing this care. Having a disabled spouse in need of care may directly influence depression risk, independently of providing this care. This is likely especially relevant for older individuals, among whom the care needs may represent a recent loss of capability. We tried to address this by examining associations restricting to respondents whose spouses had care needs. In this subgroup, caregiving was not associated with onset of depression, although effects were not precisely estimated in this much smaller sample. This result contrasts with findings from a similar study of spousal caregiving and hypertension ${ }^{10}$, where the estimated odds of hypertension onset for long-term caregiving were similar in magnitude and significance in the full sample and when restricted to respondents whose spouses had care needs. It is possible that, unlike hypertension, a caregiver's depression primarily reflects distress about the spouse's disability, rather than the consequences of caregiving per $s e$. It is important to distinguish these alternatives but more data are needed to provide sufficient statistical power to test these appropriately.

Despite these limitations, our findings have several methodological advantages and build importantly on prior research. As Pinquart and Sorensen note, many studies included in meta-analyses of caregivers' health are cross-sectional and may have inherent selection biases. ${ }^{1}$ We used a longitudinal design and adjusted for selection into caregiving duties and for sample attrition to help address endogeneity and selection bias. Our analysis depends on the assumption that we have appropriately adjusted for common causes of CG and depression. These results offer novel evidence from a nationally representative sample of older U.S. adults that do not support the notion that duration of caregiving exacerbates elevated depressive symptoms.

\section{Supplementary Material}

Refer to Web version on PubMed Central for supplementary material.

\section{Acknowledgments}

Sources of support/Acknowledgements: The authors gratefully acknowledge financial support from the American Heart Association grants 09PRE2080078 (BDC) and 10SDG2640243 (MMG) and from National Institutes of Health grant T32HD007168 (BDC). The HRS (Health and Retirement Study) is sponsored by the National Institute on Aging (grant number NIA U01AG009740) and is conducted by the University of Michigan. The authors also acknowledge David Bloom, J. Robin Moon, and three anonymous reviewers for their comments on prior versions of this paper. 


\section{References}

1. Pinquart M, Sörensen S. Differences between caregivers and noncaregivers in psychological health and physical health: A meta-analysis. Psychology and Aging. 2003; 18(2):250-267. [PubMed: 12825775]

2. Pinquart M, Sörensen S. Associations of Stressors and Uplifts of Caregiving With Caregiver Burden and Depressive Mood: A Meta-Analysis. The Journals of Gerontology Series B: Psychological Sciences and Social Sciences. 2003; 58(2):P112-P128. P112-P128.

3. Heeringa, SG. Technical description of the Asset and Health Dynamics (AHEAD) survey sample. Ann Arbor, MI: Institute for Social Research, University of Michigan; 1995.

4. Jenkins KR, Kabeto MU, Langa KM. Does caring for your spouse harm one's health? Evidence from a United States nationally-representative sample of older adults. Ageing \& Society. 2009; 29(02):277-293. [PubMed: 24567660]

5. Turvey CL, Wallace RB, Herzog R. A Revised CES-D Measure of Depressive Symptoms and a DSM-Based Measure of Major Depressive Episodes in the Elderly. International Psychogeriatrics. 1999; 11(02):139-139. [PubMed: 11475428]

6. Cole SR, Hernan MA. Constructing Inverse Probability Weights for Marginal Structural Models. American Journal of Epidemiology. 2008; 168(6):656-656. [PubMed: 18682488]

7. Haley WE, LaMonde LA, Han B, Burton AM, Schonwetter R. Predictors of Depression and Life Satisfaction Among Spousal Caregivers in Hospice: Application of a Stress Process Model. Journal of Palliative Medicine. 2003; 6(2):215-224. [PubMed: 12854938]

8. Li LW. From Caregiving to Bereavement: Trajectories of Depressive Symptoms Among Wife and Daughter Caregivers. The Journals of Gerontology Series B: Psychological Sciences and Social Sciences. 2005; 60(4):P190-P198. P190-P198.

9. Townsend A, Noelker L, Deimling G, Bass D. Longitudinal impact of interhousehold caregiving on adult children's mental health. Psychology and Aging. 1989; 4(4):393-401. [PubMed: 2515873]

10. Capistrant BD, Moon JR, Glymour MM. Spousal caregiving and incident hypertension. Am J Hypertens. 2012 Apr; 25(4):437-443. [PubMed: 22189941] 


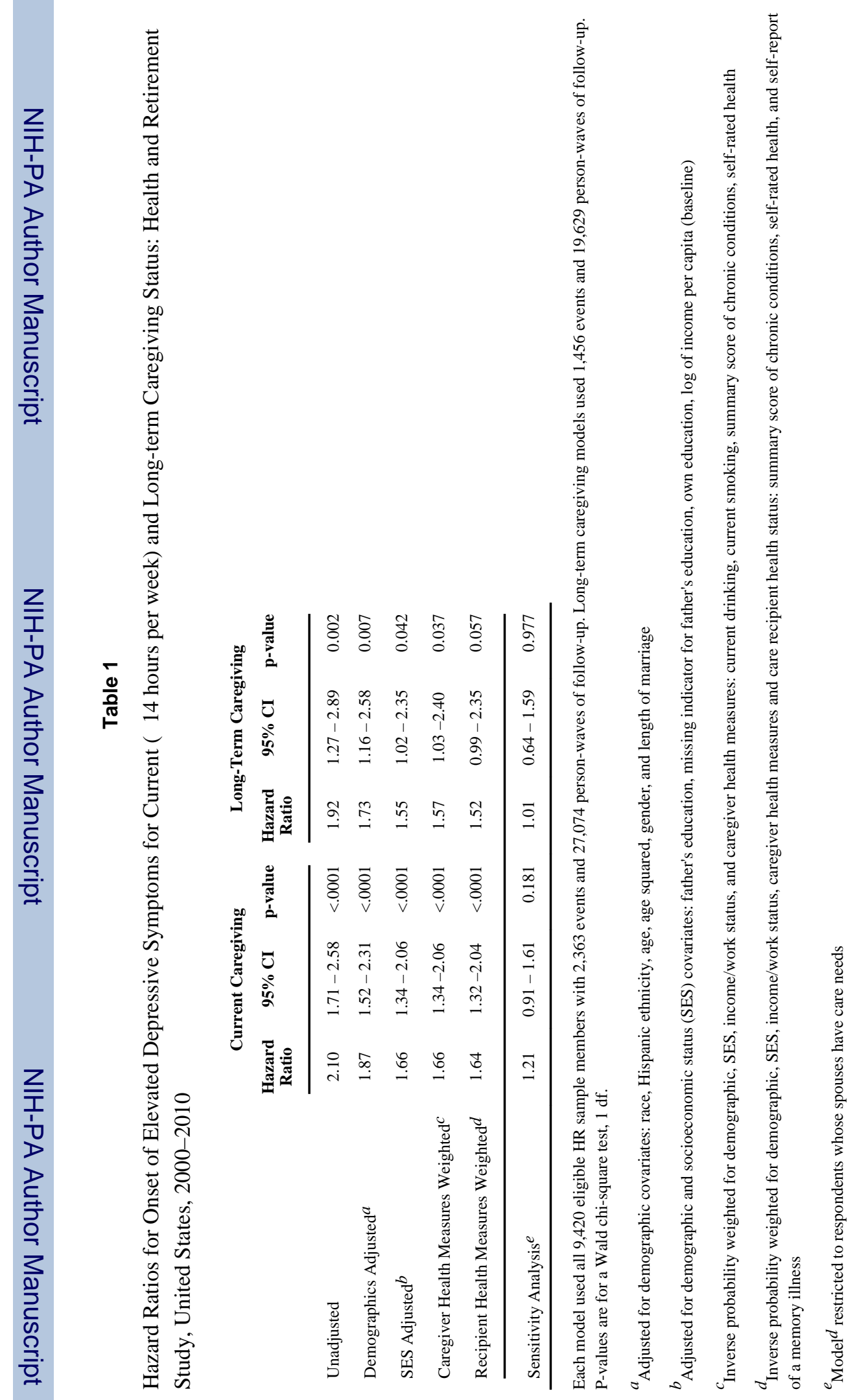

Am J Geriatr Psychiatry. Author manuscript; available in PMC 2015 August 01. 